\title{
Tapping the social psychology of psychophysical experiments: Mode of responding does not alter statistical properties of magnitude estimates
}

\author{
DANIEL ALGOM, LAWRENCE E. MARKS, and DAVID WIESENFELD \\ John B. Pierce Laboratory and Yale University, New Haven, Connecticut
}

\begin{abstract}
In an attempt to measure how mode of response might affect psychophysical judgment, 18 subjects were asked to give magnitude estimates of the loudness of $1000-\mathrm{Hz}$ tones at various sound pressure levels in each of two sessions. In one session, the subjects responded by giving their numerical judgments orally to the experimenter; in the other session, they did so by entering their judgments manually on a computer-controlled keyboard. Mode of response had no effect on the loudness function's log-log slope and a small, statistically unreliable, effect on the function's intercept.
\end{abstract}

An increasing number of psychophysical scaling studies use a fully automatic, computer-driven apparatus that requires subjects to enter their numerical responses directly on a keyboard rather than to communicate them orally to a recording experimenter. Despite the considerable resulting change in social interaction, possible effects of the new mode of responding have gone largely untested in current psychophysical research. Results of the present study, which contrasted traditional (voice) and new (keyboard) modes of responding, show that the resulting magnitude estimates remain largely, though perhaps not fully, unchanged across the procedural variation in responding.

The study of psychophysics relies as much on technological innovation as it does on substantive empirical findings and theoretical development. Ingenuity in producing and controlling stimuli restricts the set of possible attainable results. Indeed, the history of psychophysics parallels that of the instrumentation used to produce the various stimuli in sensory experiments. For example, the study of loudness, pitch, and other psychoacoustic phenomena has progressed at a pace with the technical mastery to produce an ever-increasing repertoire of auditory signals (e.g., complex spectra, extremely short durations). However, recent developments in instrumentation-in particular, the pervasive use of computers in psychophysical researchjustify examining any possible effects of such developments on responses. Computers, for instance, are used not just to produce stimuli and process data but also to directly record responses. What might be the outcome of substituting the computer for the experimenter in psychophysical research?

Of the many direct scaling methods in psychophysics, one of the most popular is magnitude estimation. Since

Preparation of this paper was supported by NIH Grant DC 00271. Address correspondence to Lawrence E. Marks, John B. Pierce Laboratory, 290 Congress Avenue, New Haven, CT 06519. its development in 1954, this method has been used in numerous applications in virtually all perceptual domains (see Gescheider, 1985, for a comprehensive summary). In addition to yielding scales of sensory magnitude, magnitude estimation can also be employed to generate equalsensation contours and to generate judgments in mixedmodality scaling. For more than three decades, magnitude estimation has served to elucidate processes of cognition and judgment as well as sensory and perceptual functioning. Its versatility for model construction, coupled with the ease of its application, has helped to make magnitude estimation one of the most widely used psychophysical methods. Much of present-day psychophysics relies on results obtained using magnitude estimation.

However, the traditional method of magnitude estimation that served so valuably in producing a rich set of fundamental results differs in one important aspect from the way in which the method is applied in an increasing number of current studies. During the first two decades of its use, in many instances magnitude estimation involved actively communicating experimenters who recorded subjects' orally given numerical responses. As in many interactive psychological experiments, there was a possibility that unintentional, covert cues might be given by experimenters. In contrast, in a growing number of modern computer-controlled studies, subjects type in their magnitude estimates and may control the rate of stimulus presentation as well as the recording of their responses. Subjects no longer interact with an experimenter during the experimental session; in most cases, no experimenter is needed. Does the change in procedure-typing the numerical estimates in solitude rather than communicating them orally to an attentive experimenter-effect changes in the resulting estimates? The question is important because if it is answered in the affirmative, we will be compelled to add yet another member to the already large set of conditions affecting judgments derived in direct scaling procedures. 
Despite the obvious change wrought by the computer revolution on social interaction in psychophysical experiments, we find no study that attempts to gauge how mode of responding might affect scaling data. Consequently, the present study addresses this issue directly by having subjects, in alternate sessions, respond orally or type in their responses to the same array of auditory stimuli.

\section{METHOD}

\section{Subjects}

Eighteen young men and women from Yale University participated in two experimental sessions conducted at least 1 day apart. None of the subjects reported a history of hearing problems, and we assumed that all had normal hearing. The subjects were paid $\$ 5$ per session.

\begin{abstract}
Apparatus and Procedure
The stimuli consisted of $1-\mathrm{sec}$ bursts of a $1000-\mathrm{Hz}$ tone, with $10-\mathrm{msec}$ rise and decay, presented monaurally to the right ear through TDH-35 headphones mounted in MX41/AR cushions. Stimuli were generated by Coulbourn Instruments modules under the control of an Apple IIe microcomputer. The tones took on 10 different sound pressure levels (40-85 dB SPL in steps of $5 \mathrm{~dB}$ ), each presented and judged a total of 10 times within the course of a session.

Each subject served in two sessions. In one they entered the responses on a keyboard, and in the other they communicated their judgments orally to an experimenter located outside the sound-isolated booth. Within a session, order of stimulus levels was random and different for every subject, with order of sessions (i.e., mode of responding) counterbalanced randomly across subjects.

The method was magnitude estimation with no specified modulus. The subjects were instructed to assign to the first stimulus whatever number they deemed appropriate to stand for its loudness, then to assign to succeeding stimuli other numbers in proportion. Whole numbers, decimals, and fractions were permitted. The number zero could reflect the absence of a sound, but it was never reported.
\end{abstract}

\section{RESULTS AND DISCUSSION}

In both response conditions, the magnitude estimates given by each subject to each stimulus were averaged geo- metrically (within sessions), and simple power functions were fitted to the relation between mean estimates and sound pressure level. Table 1 lists, for each subject, parameters of the power functions as well as other measures of performance in each condition.

The data show virtually no effect of mode of responding on performance. There was no effect on the exponent of the power function $[t(17)=1.02, p>.3]$. The voice condition provided a slightly, but insignificantly, greater intercept $[t(17)=1.54, p>.1]$. Nor is there any discernible difference in the measures of goodness of fit ( $r^{2}$ values) of the individual power functions under the two modes of responding. Clearly, having subjects enter their estimates manually onto a computer-controlled keyboard instead of communicating their judgments orally effected no significant changes in the parameters of the power functions.

We performed several other analyses of the data, but we found no evidence that mode of responding made a difference. Order of sessions mattered little to the derived exponent (a change of a mere 0.002 ), but there was an insignificant voice-first-keyboard-first difference of 0.19 in intercept. No significant differences were found in either the overall magnitude of the judgments $[t(17)=$ $0.85, p>.3$ ] or in the respective coefficients of variation between the two conditions $[t(17)=1.28, p>.2]$, although again we see the slight evidence of greater numerical estimates under the voice condition. This tendency becomes even more visible when we pool data over subjects and plot the group means (see Figure 1).

Although the difference in absolute levels is not significant, the vertical separation of the curves in Figure 1 clearly warrants further attention. Given the widespread fitting of power functions as a major analytical tool of data reduction in psychophysics, investigators may well wish to examine possible influences on the intercept. For

Table 1

Parameters of Power Function Approximations, Goodness of Fit Measures, Means, and Coefficients of Variation under the Voice and Keyboard Responding Conditions, Individually for 18 Subjects

\begin{tabular}{|c|c|c|c|c|c|c|c|c|c|c|c|c|}
\hline \multirow[b]{2}{*}{$\mathbf{S}$} & \multicolumn{6}{|c|}{ Voice } & \multicolumn{6}{|c|}{ Keyboard } \\
\hline & $\mathbf{a}$ & b & $r^{2}$ & A.M. & G.M. & C.V. & $\mathbf{a}$ & b & $r^{2}$ & A.M. & G.M. & C.V. \\
\hline 1 & -.23 & .46 & .990 & 20.9 & 15.9 & .21 & .75 & .41 & .978 & 4.4 & 3.5 & .22 \\
\hline 2 & .16 & .34 & .952 & 19.4 & 16.4 & .28 & .14 & .35 & .967 & 20.4 & 17.0 & .28 \\
\hline 3 & -.81 & .68 & .990 & 34.3 & 20.0 & .28 & -.70 & .65 & .977 & 34.5 & 20.8 & .45 \\
\hline 4 & -.18 & .37 & .951 & 11.1 & 9.1 & .36 & -.65 & .44 & .961 & 6.9 & 5.3 & .45 \\
\hline 5 & -.88 & .46 & .946 & 6.1 & 5.2 & .22 & -.68 & .32 & .981 & 4.8 & 3.7 & .31 \\
\hline 6 & -.68 & .32 & .981 & 5.9 & 4.5 & .47 & -.72 & .33 & .982 & 4.7 & 3.6 & .22 \\
\hline 7 & .23 & .32 & .946 & 20.3 & 17.4 & .18 & -.05 & .42 & .965 & 23.4 & 18.2 & .27 \\
\hline 8 & -.68 & .32 & .981 & 2.5 & 2.2 & .26 & -.72 & .33 & .982 & 2.3 & 2.0 & .18 \\
\hline 9 & -.41 & .32 & .988 & 4.5 & 3.9 & .19 & -.66 & .38 & .992 & 3.9 & 3.3 & .22 \\
\hline 10 & -.20 & .69 & .974 & 149.8 & 88.0 & .36 & -.20 & .70 & .950 & 160.8 & 94.6 & .33 \\
\hline 11 & -.48 & .37 & .947 & 6.0 & 6.1 & .32 & -.63 & .48 & .976 & 5.3 & 4.2 & .31 \\
\hline 12 & -.68 & .46 & .963 & 8.2 & 5.9 & .32 & -.52 & .44 & .996 & 9.1 & 7.1 & .35 \\
\hline 13 & -.98 & .41 & .961 & 24.0 & 18.9 & .32 & .13 & .39 & .982 & 27.6 & 22.6 & .33 \\
\hline 14 & .25 & .40 & .990 & 38.0 & 31.1 & .21 & .15 & .39 & .994 & 29.4 & 24.0 & .26 \\
\hline 15 & -1.00 & .47 & .953 & 13.4 & 11.8 & .22 & -.90 & .44 & .971 & 9.1 & 7.4 & .30 \\
\hline 16 & .79 & .20 & .917 & 41.4 & 31.9 & .33 & 1.10 & .21 & .922 & 38.3 & 30.1 & .44 \\
\hline 17 & -1.00 & .47 & .953 & 3.9 & 3.0 & .19 & -.90 & .44 & .971 & 3.9 & 3.0 & .19 \\
\hline 18 & .23 & .20 & .956 & 7.4 & 6.9 & .12 & -.04 & .28 & .971 & 7.9 & 7.1 & .22 \\
\hline
\end{tabular}

Note-S = subject, $\mathrm{a}=$ intercept of power function, $\mathrm{b}=$ exponent of power function, $r^{2}=$ goodness of fit, A.M. = arithmetic average of magnitude estimates, G.M. = geometric average of magnitude estimates, C.V. = median coefficient of variation of magnitude estimates. 


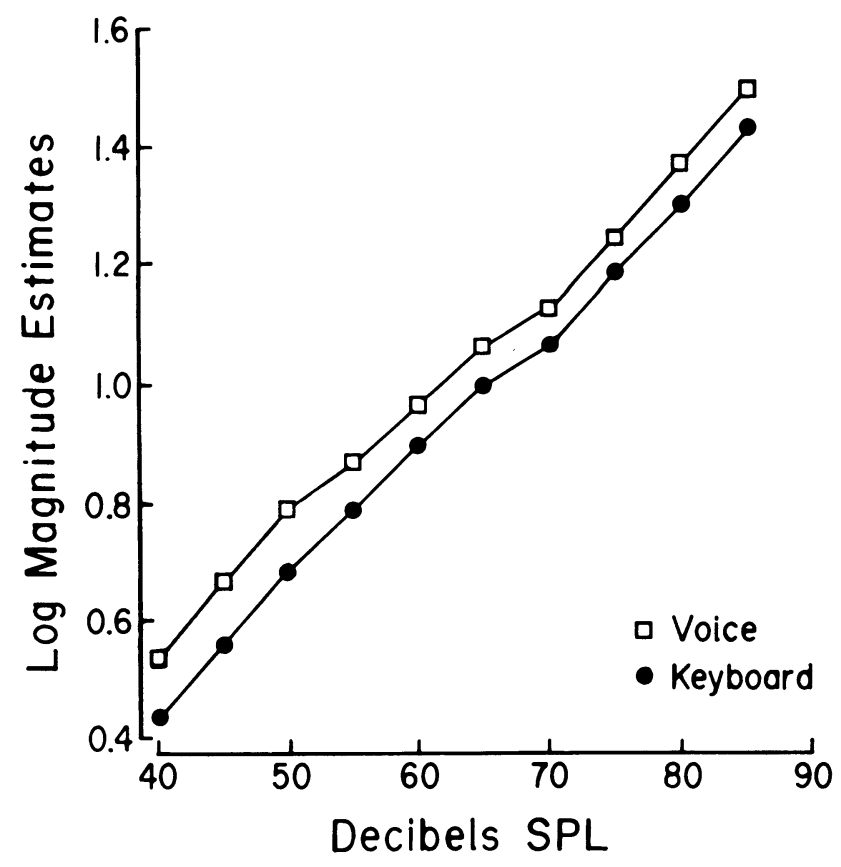

Figure 1. Average loudness functions for voice and keyboard modes of responding. example, some theories, notably that of Zwislocki (1983; see also Zwislocki \& Goodman, 1980), postulate an "absolute" coupling between stimulus intensities and individual numerical responses. Inferences about absolute scaling may require modification should the sizes of the numbers depend on the mode of responding.

In summary, this study sought to provide comparative data on the way that two widely used methods of responding might influence the statistical properties of magnitude estimates. The results of the present experiment provide some badly needed preliminary evidence that the increased use of subject-controlled direct feeding of data does not alter to any substantial degree the important properties of those data. This may provide some relief for investigators who use fully automated, computer-driven experiments in which subjects generate magnitude estimates.

\section{REFERENCES}

Gescheider, G. A. (1985). Psychophysics: Method, theory, and application (2nd ed.). Hillsdale, NJ: Erlbaum.

ZWISLOCKI, J. J. (1983). Group and individual relations between sensation magnitudes and their numerical estimates. Perception \& Psychophysics, 33, 460-468.

ZwislockI, J. J., \& Goodman, D. A. (1980). Absolute scaling of sensory magnitudes: A validation. Perception \& Psychophysics, 28, 28-38. 